\title{
Sizing Particles in Gases by an Advanced Optical Procedure
}

\author{
Dan Chicea ${ }^{1,2, *}$, Cristian Leca ${ }^{1,2}$, and Sorin Olaru ${ }^{1,2}$ \\ ${ }^{1}$ Faculty of Sciences, University: Lucian Blaga, Street: Dr. Ion Raţiu 5-7, Sibiu city, Romania \\ ${ }^{2}$ Research Center for Complex Physical Systems, Dr. Ion Ratiu 5-7, Sibiu city, ROMANIA, 550012
}

\begin{abstract}
Several types of sensors capable of detecting fumes are commercially available. A well-established procedure for sizing particles suspended in liquid solvent is called Dynamic Light Scattering (DLS) and is based on assessing the diffusion coefficient of the particles from the fluctuations of the scattered light recorded in the scattered light intensity time series. This work highlights the difficulties encountered in an attempt to use DLS for sizing particles suspended in air and the particular, narrow experimental conditions chosen in designing and setting such a DLS experiment. The experimental setup was tested on combustion gases and the results of the preliminary experiment are presented in detail. The results stand as a proof of concept for using DLS in sizing particle in gases and in designing a sensor for fumes detection that can indicate the average size of the particles over an extended range.
\end{abstract}

\section{Introduction}

As the Laser beam is pointed to particles dispersed in the carrier fluid, each illuminated particle disperses light so that the particle becomes a light source. Particles in suspension act as dispersion centers or light scattering centers (referred to as SCs). The incident light is coherent (emitted from a Laser), therefore the emitted waves produce an interference field. But suspended particles are moving continuously due to Brownian motion. The consequence is the aspect of random position and intensity of the maxima on the interference field, which gave it the name of "boiling speckles".

The Dynamic Light Scattering (DLS) is the method based on the dependency between the chaotic motion of the particles and the dynamics of the interference field. The basic principle of this method or procedure is described in many works, only a few of them are stated [1-4].

The most important applications of DLS are to analyze the size distributions and scale of nanoparticles, colloids and proteins in suspensions in various solvents [5]. The DLS methodology turned out to be appealing [6] as it has some benefits over other experimental approaches, one of them relaying on the ability of producing absolute, without calibration being required.

This paper is in line with the results reported in [7], which highlights the findings of the theoretical study on the possibility of applying DLS method on samples that have air as a susspension fluid. Measurements on the size of the particles in fumes from burned wax are presented and compared with the literature. Details are presented further on.

\footnotetext{
*Corresponding author: dan.chicea@ulbsibiu.ro
} 


\section{The DLS procedure}

The DLS experimental setup comprises a coherent light source, the target, a detector and a data acquisition system (DAS hereafter) connected to a computer for recording and processing the recording. The coherent light source was chosen to be a Laser diode, for keeping the setup as simple as possible. The wavelength is a typical red light of $633 \mathrm{~nm}$ and a small scattering angle $\theta$ was chosen, as described in [7], in order to cover the frequency spectrum beyond the roll-over point, as we will point out in Section 3 .

The scattering angle $\theta$ was set to $5^{\circ}$, according to the restricted conditions required by air as solvent, in line with the findings reported in [7]. The burned gases temperature was $53^{\circ} \mathrm{C}$ in the tube and it was measured using an infrared thermometer. The gases that were the target of the laser beam were produced by burning wax. The cuvette-detector distance D used in this work was $0.1 \mathrm{~m}$.

The arrangement of the experiment for DLS in air is presented in Fig. 1.

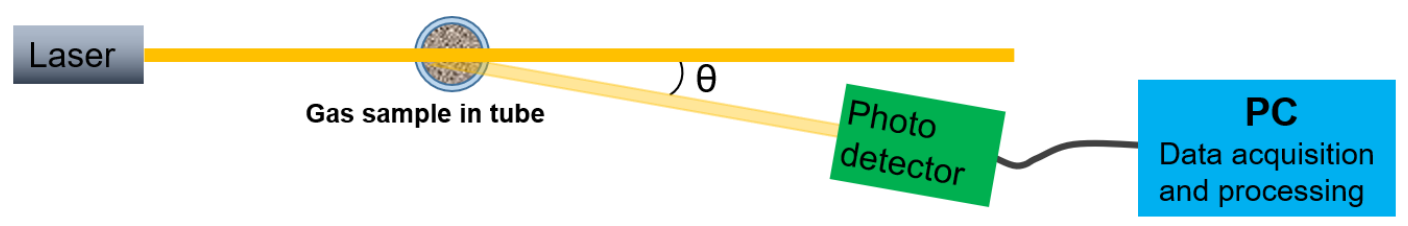

Fig. 1. The experimental setup (simplified schematic) for DLS in air.

A detector at angle $\theta$ is used to measure the light intensity. The signal is digitized with a data acquisition system (DAS hereafter) and the resulting DLS time series is recorded. The time series is a set of digital values recorded by the DAS with a constant sampling rate $\mathrm{f}$. That is, analog to digital conversions are done at equal time intervals $\Delta \mathrm{t}=1 / \mathrm{f}$. According to $[2,8,9]$, the diffusion coefficient is proportional to the width of the autocorrelation function resulted from the time series.

The early reporting of $[1,10]$ and the following theoretical improvement [11-13], revealed a relation between the frequency spectrum (hereafter FS) of the intensity of scattered light and the probability density function (PDF). The frequency spectrum resulted from the experimental data can match the Lorentzian line S(f) (1) [14, 15]:

$$
S(f)=\frac{a_{0} \cdot a_{1}}{(2 \pi f)^{2}+a_{1}^{2}}
$$

$\mathrm{S}(\mathrm{f})$ is determined by two parameters $\mathrm{a}_{0}$ and $\mathrm{a}_{1}$. The optimum values of $\mathrm{a}_{0}$ and $\mathrm{a}_{1}$ can be found using the method least square fit minimization for the best match of the frequency spectrum to Lorentzian line $\mathrm{S}(\mathrm{f})[14,15]$. The radius of particles can be calculated from the following equations (2) and (3):

where:

$$
R=\frac{2 k_{B} T q^{2}}{6 \pi \eta a_{1}}
$$

$$
\mathrm{q}=\frac{4 \pi \mathrm{n}}{\lambda} \sin \frac{\theta}{2}
$$

In the above equations (2) and (3), $\mathrm{R}$ is the average radius of particles in suspension, $\mathrm{k}_{\mathrm{B}}$ is Boltzmann's constant, $T$ is the absolute temperature of suspension, $\eta$ is the (dynamic) viscosity coefficient of the solvent, $\mathrm{q}$ is the wave scattering vector, $\mathrm{n}$ is the solvent refractive index, $\lambda$ is the wavelength of the laser light and $\theta$ is the scattering angle $[14,15]$. All quantities in equations (1)(3) are expressed in International System of Units. 
One can observe from equation (2) that for a given radius $\mathrm{R}$ of the particles, $\mathrm{a}_{1}$ parameter is inversely proportional to the viscosity $\eta$. So that a decrease of $\eta$ will result in the increase of $a_{1}$ parameter, shifting the turnover point in the frequency spectrum graph towards higher frequency, as shown in Fig. 2. If the standard value of $90^{\circ}$ is maintained for $\theta$, which is the scattering angle, then a higher data acquisition sampling rate $\mathrm{f}$ is required. This alternative will require more expensive data acquisition systems. By choosing this alternative we remain in the area of keeping a low-cost for the device, using conventional rather than custom designed electronics.

In order to remain in line with the simulation results in [7] a low-cost audio preamplifier was chosen and assembled from a kit. The amplification of the preamplifier was investigated to assess the relatively constant plateau, and, consequently, to choose the data acquisition sampling rate. The sampling rate was chosen to be $\mathrm{fs}=16 \mathrm{kHz}$. For this reason, the scattering angle to perform DLS time series recording was selected to be very small, that is $5^{\circ}$. Using a small scattering angle allows the rollover point in the log-log plot of the FS of the scattered light intensity to lay within the frequency range. This feature is crucial for assessing the $a_{1}$ parameter in eq. (2) and therefore in calculating the diameter in eq. (3).

Moreover, scattering at very small angles is done with bigger intensity than at bigger angles, as pointed out in [16] and [17]. A bigger scattered light intensity is quite mandatory when attempting to do DLS with air as solvent, because the particles concentration in fumes is much smaller than a typical concentration in water. In order to process the DLS time series, a good signal to noise ratio is required, therefore an intense scattered light intensity is mandatory and a small scattering angle for recording fits very well in this purpose.

\section{Results and discussion}

The schematic of the device is presented in Fig. 1. A wick impregnated with natural wax was burned underneath the tube and a small funnel was used both to concentrate the burned gases and to ensure that ascending gases do not cross the laser beam other than through the glass tube. In this manner the scattering angle could be measured precisely and was $5^{\circ} \mathrm{C}$. Scattered light time series were acquired at an acquisition rate $\mathrm{fs}=16 \mathrm{KHz}$ and the temperature of the gases through the tube, after reaching a dynamical thermal equilibrium, was $57^{\circ} \mathrm{C}$.

Air viscosity was calculated using the Sutherland correlation [18], which expresses a relationship between the dynamic viscosity and the absolute temperature of an ideal gas, based on kinetic theory of ideal gases.

Each time series lasted for 5 seconds. The PS was calculated using the fast Fourier transform algorithm [19] (FFT) and only $2^{\wedge} 16=65536$ data were used. The reason for this selection is that the FFT algorithm uses a number of data of the type $2^{\mathrm{n}}$. If more data is supplied, the function fills the rest of the values with 0 s to match the number $2^{(n+1)}$, with a direct consequence of indicating a much bigger amplitude for very small frequencies that the real one.

A PS of one of the recorded DLS time series is presented in Fig. 2. We notice from the beginning that the rollover point is in the range $10-30 \mathrm{~Hz}$, which enables assessing $\mathrm{a}_{1}$ and therefrom the particles diameter. We also notice that there does exist strong noise in the spectrum, illustrated by the dots indicating amplitudes much bigger than the general grouping of them.

A filtering procedure was used after the PS was computed, as in [20-22], that removed a window of $2 \mathrm{~Hz}$ width centered on $50 \mathrm{~Hz}$ frequency and the upper harmonics, as $50 \mathrm{~Hz}$ is the power grid frequency and such noise is ubiquitous. 


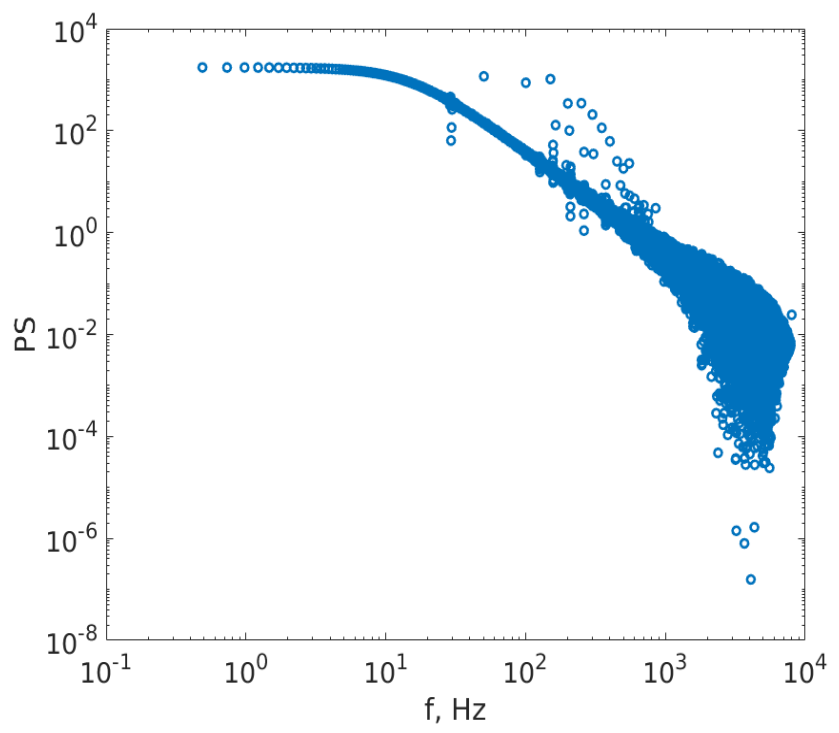

Fig. 2. The Power spectrum (PS) of a DLS time series recorded in air at $5^{\circ}$.

After filtering the FS spectrum, the Lorentzian line was fit to the spectrum as mentioned in Section 2, $a_{0}$ and $a_{1}$ were determined and, there from, radii of the particles using eq. (2). Fig. 3 depicts the PS after filtering (the circles) and the Lorentzian line (the continuous line). We notice that some of the noise frequencies were removed, but other big amplitudes remained. They might be produced by the power supply of the Laser diode used as coherent light source. Further improvement of the filtering procedure is in progress.

The $\mathrm{a}_{1}$ parameter was $94.21 \mathrm{~Hz}$, therefore the average diameter of the particles in fume was $0.387 \mu \mathrm{m}$.

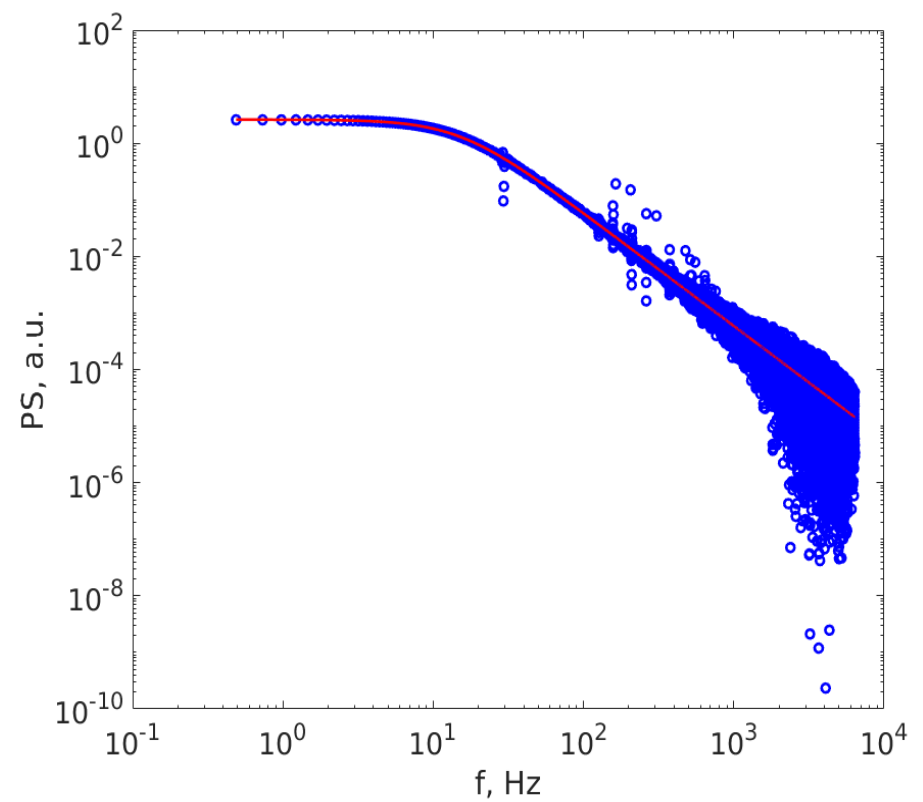

Fig. 3. The PS of the DLS time series after filtering (circles) and the Lorentzian line (continuous red line). 


\section{Conclusion}

This work is a continuation of the simulation work reported in [7], which proved that, theoretically, DLS is possible on air as solvent. In order to remain in the range of a low-cost experimental device, aiming to produce a prototype, a standard audio preamplifier was used, coupled with a phototransistor as detector. As suggested in [7] the scattering angle was as small as $5^{\circ}$, the data acquisition frequency was $16 \mathrm{kHz}$ and the data processing procedure was the same as in previous work $[14,15,20,21]$. The procedure presents systematic errors, as the very small angle relatively big error in measuring and the frequency range is small as compared with commercially available devices. The average size of the particles produced by burning wax through a wick was found to be $0.387 \mu \mathrm{m}$, a size which is in good agreement with the literature concerning particle emissions in fumes [23].

Nevertheless, the simple, low-cost experimental setup and the data processing procedure represent a proof of concept for extending DLS to suspensions in air as a procedure for sizing particles in fumes and exhaust gases.

\section{References}

1. N.A. Clark, J.H. Lunacek, G.B. Benedek, AM J PHYS, 38, 575 (1970)

2. J.W. Goodman, Laser speckle and related phenomena (Springer, Berlin, 1975)

3. H.C.v.d. Hulst, Light scattering by small particles (Courier Corporation, New York, 1981)

4. R. Xu, Particle characterization: Light scattering methods (Springer, Netherlands, 2002)

5. S. Bhattacharjee, J CONTROL RELEASE, 235, 337 (2016)

6. J. Stetefeld, S.A. McKenna, T.R. Patel. Biophysical reviews, 8 (4), 409 (2016)

7. D. Chicea, C. Leca, BRCEBE 2019, Investigation on the Possibility of Designing an Educational Dynamic Light Scattering Device for Sizing Particles Suspended in Air, (Sibiu, Romania, 2019)

8. W. Tscharnuter, Encyclopedia of Analytical Chemistry (John Wiley \& Sons, Hoboken-New Jersey, 2006)

9. B.B. Weiner, Liquid and Surface Borne Particle Measurement Handbook (Taylor \& Francis, New York, 1996)

10. S.B. Dubin, J.H. Lunacek, G.B. Benedek, Proceedings of the National Academy of Sciences of the United States of America, 57, 1164 (1967)

11. B.J. Berne, R. Pecora, Dynamic light scattering: with applications to chemistry, biology, and physics (John Wiley \& Sons, USA, 2000)

12. J.W. Goodman, Statistical Optics-Wiley Classics Library (Wiley, New York, 2000)

13. E. Hecht, Optics (Addison-Wesley Publishing Company, 2001)

14. D. Chicea, CURR NANOSCI, 8, 259-65 (2012)

15. D. Chicea, E. Indrea, C. Cretu, J OPTOELECTRON ADV M, 14, 460-6 (2012)

16. D. Chicea, I. Turcu, OPTIK, 118, 232-6 (2007)

17. D. Chicea, APPL OPTICS, 47, 1434-42 (2008)

18. W. Sutherland, The London, Edinburgh, and Dublin Philosophical Magazine and Journal of Science, 36, 507 (1893)

19. J.W. Cooley, J.W. Tukey, MATH COMPUT, 19, 297 (1965)

20. D. Chicea, MEAS SCI TECHNOL, 28, 055206 (2017)

21. D. Chicea, S.M. Rei, MEAS SCI TECHNOL, 29, 105201 (2018)

22. D. Chicea, Sensors, 20, 3425 (2020)

23. https://www.coloradoci.com/bin-pdf/5270/ParticleSize.pdf, [Accessed at 31.03.2021] 